\title{
Assessing driver's mental representation of Adaptive Cruise Control (ACC) and its pos- sible effects on behavioural adaptations
}

\author{
Giulio Francesco Piccinini ${ }^{\mathrm{a}^{*}}$, Anabela Simões ${ }^{\mathrm{b}}$, Carlos Manuel Rodrigues $^{\mathrm{c}}$ and Miguel Leitão ${ }^{\mathrm{d}}$ \\ ${ }^{a}$ UNIVERSITAS, Cooperativa de Ensino Superior e Investigação Cientifica C.R.L., Alameda das Linhas de Torres \\ 179, 1750-142, Lisboa, PORTUGAL \\ ${ }^{\mathrm{b}}$ CIGEST, Instituto Superior de Gestão, Rua Vitorino Nemésio 5, 1750-306, Lisboa, PORTUGAL \\ ${ }^{\mathrm{c}}$ Departamento de Engenharia Civil, Faculdade de Engenharia da Universidade do Porto, Rua Dr. Roberto Frias \\ $s / n, 4200-465$, Porto, PORTUGAL \\ ${ }^{\mathrm{d} D e p a r t a m e n t o ~ d e ~ E n g e n h a r i a ~ E l e c t r o t e ́ c n i c a, ~ I n s t i t u t o ~ S u p e r i o r ~ d e ~ E n g e n h a r i a ~ d o ~ P o r t o, ~ I n s t i t u t o ~ P o l i t e ́ c n i c o ~ d o ~}$ \\ Porto, Rua Dr. António Bernardino de Almeida 431, 4200-072, Porto, PORTUGAL
}

\begin{abstract}
The introduction of Adaptive Cruise Control (ACC) could be very helpful for making the longitudinal driving task more comfortable for the drivers and, as a consequence, it could have a global beneficial effect on road safety. However, before or during the usage of the device, due to several reasons, drivers might generate in their mind incomplete or flawed mental representations about the fundamental operation principles of ACC; hence, the resulting usage of the device might be improper, negatively affecting the human-machine interaction and cooperation and, in some cases, leading to negative behavioural adaptations to the system that might neutralise the desirable positive effects on road safety. Within this context, this paper will introduce the methodology which has been developed in order to analyse in detail the topic and foresee, in the future, adequate actions for the recovery of inaccurate mental representations of the system.
\end{abstract}

Keywords: ADAS; ACC; behavioural adaptation; mental representation; road safety

\section{Introduction}

Road accidents are the cause of death for nearly 1.3 million people every year and, unless immediate and effective action is taken, road traffic injuries are predicted to become the fifth leading cause of death in the world, resulting in an estimated 2.4 million people killed each year [21]; such a high number of accidents is brought about by the extreme dynamism of the road system which is dominated by uncertainty so that any strategy, which is elaborated to challenge the multi-dimensional issue of road accidents, might not encounter the expected success. However, despite the intrinsic uncertainty, the occurrence of a traffic crash is not the consequence of the case, but the ef- fect of a chain of events, resulting from the interaction of the three critical elements of the road system, the road user (driver, pedestrian, etc.), the vehicle and the environment. Then, some actions could be taken to avoid road accidents (before they occur) and to mitigate their effects (once they happened). Regarding the mitigation of the effects, as it is widely recognized by the road safety community, the massive introduction of passive safety measures in vehicles (seat belts, airbags, crumple zone, etc.) highly contributed to the reduction of fatalities which we have seen in Europe, United States and Japan in the last 2 decades. On the other hand, it appears that, in the coming years, the margin of improvement for those crash effects reduction measures is very small and, therefore, the trend in vehicle safety is moving from

\footnotetext{
${ }^{*}$ Corresponding author. Tel.: +351217541310. E-mail address: g.f.piccinini@gmail.com
} 
crash mitigation to crash prevention, through the adoption of various measures polarized on the road user (e.g., education and training), the vehicle (e.g., active safety devices) and the environment (e.g., road infrastructure design). Among the crash preventive actions concentrated on the vehicle, technologies as Advanced Driver Assistance Systems (ADAS) have the potential to play a considerable role for the improvement of traffic safety in the coming years [5].

\subsection{Advanced Driver Assistance Systems (ADAS)}

ADAS are assistance systems supporting the drivers in the primary driving task, referring with that to the activities undertaken by the driver to maintain the longitudinal and lateral control of vehicles within the traffic environment; these devices actively stabilize or manoeuvre the car, but without taking over the task completely, leaving the responsibility always to the driver [12].

The acronym ADAS includes devices providing different sorts of assistance and a possible classification can be made according to the typology of control which they supply, dividing them into two categories.

- Lateral control: systems supporting the driver in keeping the correct lateral behaviour such as Lane Change Assistant (LCA), Lane Departure Warning (LDW) and Blind Spot Information System (BLIS).

- Longitudinal control: devices supporting the driver in maintaining the correct longitudinal behaviour, including Adaptive Cruise Control (ACC), Forward Collision Warning (FCW) and Intelligent Speed Adaptation (ISA).

In this paper, the focus will be put on a specific ADAS: Adaptive Cruise Control.

\subsection{Adaptive Cruise Control (ACC)}

Adaptive Cruise Control (ACC), known also as Advanced/Autonomous/Intelligent Cruise Control, is a comfort and convenience system introduced, as an extension of conventional cruise control, firstly in Japan in the late 90's and, afterwards, in Europe and United States. Using ACC, the driver can set both the desired speed and the headway to the preceding vehicle; in response to those settings, the system will adjust speed and headway by controlling fuel flow or by slightly braking, detecting the driver ahead through the frontal radar/laser sensor [19]. When ACC is working, if critical situations arise (like the overtaking of another vehicle or an emergency brake), the driver can over-ride the system, at any time, by pressing the accelerator pedal or, alternatively, he/she can temporarily deactivate the system by pushing the ACC activation/deactivation button, by depressing the brake, or by pressing for a long period the accelerator or the clutch pedal.

In the last years, car makers have undertaken technological developments in order to bring to the markets new versions of Adaptive Cruise Control; the one considered as the next generation of the traditional ACC is the "Stop\&Go" system, which allows the vehicle to slow down up to a complete standstill. To do this, "Stop and go" ACC, among other things, has to be capable of detecting other road users or stationary objects at a much closer range than the common ACC [19].

Despite the undoubted assistance that ACC can provide to the users, the device has not been marketed as safety systems but, rather, as "Comfort and driving pleasure" technology in order to emphasize that the driver is, nevertheless, the ultimate responsible for the driving activity. However, through this device, a crucial modification has been introduced. The relationship between human and machine has evolved from a simple human-machine interaction (HMI), in which the user fully controls the system, onto a more complex and dynamic context, in which the machine processes information and the user assumes a supervisory role [6]. Then, although ACC might help the human in keeping the longitudinal control of the vehicle, there is also the possibility that drivers might use the system in unexpected ways, compromising road safety. It is relevant to notice that, during the driving with ACC, not only the driving performances (braking, steering, etc.) will be modified but also, other aspects of the driving task will be affected, so that it should be assumed that trade-offs of mobility for safety are possible [16]. One critical example of those trade-offs is the occurrence of drivers' negative behavioural adaptation to the system.

\subsection{Behavioural adaptation}

Adaptation, in biology, can be defined as the process of change by which an organism or species becomes better suited to its environment and, therefore, able to survive to the rising pressures and opportunities; in social evolutionism, a similar approach has been adopted, with cultural innovations seen as the objects of environmental selection and the means through which social groups may be able to adapt to their physical and social environment. Then, from 
these definitions, it is clear that adaptation should be regarded as a manifestation of intelligent behaviour.

In the context of road safety, describing the human-machine interaction, the Organization for Economic Co-operation and Development (OECD) defined 'behavioural adaptation' as 'those behaviours which might occur following the introduction of changes to the road-vehicle-user system and which were not intended by the initiators of the change. [...] They create a continuum of effects ranging from positive increase in safety to a decrease in safety" [10]. Saad, in [15], stated that those behavioural changes could appear within the very activity of the equipped drivers or within their interaction with other road users and they can evolve from the complex interplay of different factors:

a) Specific features of the assistance systems.

b) Variability of the driving situations.

c) Individual driver characteristics.

Extremely tied with those three factors is the process of learning with whom the driver takes possession of the purpose, working principles and expected performance of the ADAS he/she interacts with. This will direct affect the usage which the driver makes of the system. Notably, we speak about the temporal factors affecting behavioural adaptation and two main phases are, namely, considered [3]:

- 'Learning and appropriation' phase: during this period, the driver discovers the system, learns how it operates and identifies its limits. This learning process is assumed to be crucial for the driver's mental representation of the system, the confidence he/she has in it and its optimal use.

- 'Integration' phase: the driver, through experience using the system in different road situations, reorganises his/her activity by integrating the system in the management of the overall driving task.

The deep analysis of the 'learning and appropriation' phase is relevant because, depending on its progress and duration, it is expected a different evolution over time of the drivers' behaviour and, as a consequence, a different moment for the insurgence of short, medium or long-term behavioural adaptations to ADAS.

\subsection{Mental representation of the system}

As already mentioned in the previous section, strictly linked to the 'learning and appropriation' phase is the drivers' shaping of mental representations (or mental model) of the systems; as defined by Rouse and Morris, "mental models are the mechanisms whereby humans are able to generate descrip- isms whereby humans are able to generate descriptions of system purpose and form, explanations of system functioning and observed system states, and predictions of future states" [13]. Then, it is clear that mental representations/models directly influence the interaction and the cooperation between the drivers and the system because they reflect users' opinions about the characteristics (functioning principles, usage conditions, etc) of the device. Unfortunately, mental representations/models are interiorised models, formed in a specific context and for a specific aim. As a consequence, they are not true copies of the objective reality, but they might diverge from it considerably; therefore, when they are not properly created, they can trigger misuses of the system, leading to possible dangerous situations.

Research on mental models/representations has previously been carried out, in Human Factors, for understanding human behaviour in the interaction with machines (e.g., [4], [11] and [20]). On the other hand, in Traffic Psychology, researches aimed to better comprehend the processes leading a driver to evaluate a certain driving situation and, as a result, to take a specific action (e.g., [2] and [9]).

\section{Objective}

Several researches have been conducted to study drivers' behavioural adaptations in response to ACC (e.g., [7], [14] and [17]) through the measurement of different variables (speed, headway to the vehicle in front, workload, lane keeping, etc.). However, the findings collected so far are not always converging and, therefore, concerning the behavioural adaptations to the ACC, it is not possible yet to make a clear evaluation about the positive or negative effects of the system on road safety. Furthermore, to the knowledge of the authors, it has been rarely studied the mental representation of ACC (e.g., [8] and [18]) despite this research topic might deserve some more attention; indeed, the accuracy of the drivers' mental representation of the system will influence the usage that people make of the device and, in case the mental representation is not appropriate, some disruption could occur in the human-machine interaction and cooperation, possibly affecting in a negative way the road safety.

As a first objective, therefore, this research will try to uncover the progress and length of the "learning and appropriation" phase for ACC, investigating how 
the drivers' mental representation of the system evolves with the usage of the device.

Then, it will aim at discovering how the mental representation that the drivers build about ACC might influence the usage made of the system by people. Especially, it will be researched if any behavioural adaptations could be caused by a wrong mental representation of the system.

Finally, in case a correlation is found between the improper usage of the system and an erroneous mental representation, it will be suggested a series of actions to find a solution for the issue.

In the lines below, the objectives are listed in a more structured way:

1. measure the drivers' mental representation of ACC and its evolution during the usage of the system (especially, short and medium term usage);

2. detect the positive and negative behavioural adaptations which occur during the short and medium term usage of the system;

3 . in case negative behavioural adaptations to ACC are caused by erroneous mental representation of the system, propose some actions in order to avoid that the same trouble can appear with future customers.

\section{Method}

Given the objectives listed in the previous section, this research is focalised on two main steps: measuring the drivers' mental representation of ACC and identifying possible behavioural adaptations to the system.

Concerning the first aspect, various methodologies have been used in the past to study mental representation of systems or tasks (protocol analysis, concept sorting, interviews, etc.) and, after a bibliography review on the topic, it was finally decided for this study to adopt a technique which was inspired by the reading of Bailly et al. [1] and based on the presentation of scenarios during an interview.

On the other hand, regarding the second aspect, it was decided to investigate behavioural adaptations to ACC studying the driver behaviour when he/she interacts with the system in a real driving setting. In order to achieve that, the performance of a field operational test (FOT) has been planned because, compared to other methods (e.g. simulator studies), it allows higher degree of validity.
In the next sections, both methods will be defined in more detail.

\subsection{Interviews with scenario presentation}

In this study, in order to measure the drivers' mental representation of $\mathrm{ACC}$, it has been considered to use interviews where some ad-hoc scenarios will be presented to the participants; in more detail, those scenarios will show driving situations which might occur to the participants when driving with ACC active. The adoption of such scenarios should guarantee to the research team to quantitatively measure the mental representation of the system that drivers built; then, it will be possible the comparison of results between different participants and, for the same participant, between different moments in which the scenarios are presented.

As explained previously, the scope of the research is not only to measure the drivers' mental representation of ACC but also, to determine the progress and length of the "learning and appropriation" phase for the system. This will be attempted through the investigation of how the mental representation of ACC evolves in the short and medium term usage of the system. Therefore, the interviews (presentation of scenarios) with the participants should be carried out more than once in order to describe the progress of the mental representation of the system; this will be done during the performance of the Field Operational Test as explained in the next section.

\subsection{Field Operational Test (FOT)}

The Field Operational Test is a recent technique mainly used in studies that aim at evaluating a specific in-vehicle system in environmental conditions that are similar to the ones that participants encounter in his/her normal driving task; in the recent years, FOT have been already used to investigate the effects of both In-Vehicle Information Systems (IVIS) and ADAS use on driver behaviour. Compared to Naturalistic Driving Study, FOT allows to the research team a higher level of control on the experiment because it is possible to give some instructions to the participants about the usage of the system.

In the case of this research project, the FOT has the main scope of evaluating, in a real setting, short and medium term drivers' behavioural adaptations to ACC. In order to perform that, it is planned to carry out the FOT using fleet vehicles and recruiting participants who are experienced drivers, but that never 
used the system (this last request is important to see the progress of ACC usage since the beginning). However, the sample of participants taking part in both the interviews and FOT will be quite small (around 10 individuals).

The vehicle used for the experiment will be equipped with cameras, accelerometers and sensors in order to get the data required for the study. The FOT will be carried out for a time of 1 month for each driver and will follow a specific schedule.

1. In the first week of vehicle's usage, the driver won't be allowed to use ACC and this recording will be used as an "antes" baseline for further comparison.

2. After the first week, drivers can use the systems and the 1-month observation officially starts.

3. After the month of observation, the participants will be asked again to drive for a week without using the ACC ("post" baseline).

The usage of an "antes" and a "post" baseline will allow the comparison of the driver's behaviour before and after using the ACC to see if any behavioural adaptations, caused by the system, occurred.

As mentioned in the previous section, during the performance of the FOT, interviews will be carried out in different moments in order to describe the progress of drivers' mental representation during the usage of the system.

A first interview will be conducted after the first 3 days of usage of the system (after the first week of "antes" baseline"). A second interview will be carried out after the first 2 weeks of driving with the system. Finally, a third interview will be performed at the end of the FOT (after the "post" baseline).

\section{Expected results and next steps}

This paper presented a methodology to measure the drivers' mental representation of ACC and its influence on behavioural adaptations to the device. According to the current schedule (drafted in August 2011), the study is planned to begin in the next months (October or November 2011) and it will last for around 6 months in order to end before June 2012.

\section{Limitations of the study}

The outlined inquiry strategy presents clear limitations due to some constraints (mainly, limited time and restricted budget).
As a first limitation, it is evident that the small number of participants (both in the interviews and in the FOT) will not allow, as a result of the data analysis, any statistical generalization, restricting the dimension of the research to a pilot study to be validated by other future works.

As a second limitation, it should be considered the usage of scenarios to measure the drivers' mental representation of ACC; indeed, such technique has been specifically developed for this study and never been tested before.

Finally, the usage of a FOT to identify phenomena of behavioural adaptations might not be successful due to the low level of experimental control typical of this methodology (compared to driving simulator studies).

\section{References}

[1] B. Bailly, T. Bellet and C. Goupil, Driver's mental representations: Experimental study and training perspectives, Proceedings of the First International Conference on Driver Behaviour and Training, 2003.

[2] T. Bellet, B. Bailly-Asuni, P. Mayenobe and A. Banet. A theoretical and methodological framework for studying and modelling driver's mental representations, Safety Science 47 (2009), pp.1205-1221.

[3] P.C. Cacciabue and F. Saad,. Behavioural adaptations to driver support systems: a modelling and road safety perspective, Cognitive, Technology \& Work (2008) 10(1), pp. 31-39.

[4] J.K. Doyle and D.N. Ford, Mental models concepts for system dynamics research, System Dynamics Review, 14, 3-29, 1998.

[5] European Commission, Towards a European road safety area: policy orientations on road safety 2011-2020, European Commission Communication COM(2010) 389 final.

[6] J. Hoc, From human-machine interaction to human-machine cooperation, Ergonomics 43(7), 833-843 (2000).

[7] M. Hoedemaeker and K.A Brookhuis, Behavioural adaptation to driving with an adaptive cruise control (ACC), Transportation Research Part F 1 (1998), pp. 31-39.

[8] T.A. Kazi, N.A. Stanton, G.H. Walker and M.S. Young, Designing driving: driver's conceptual models and level of trust in Adaptive Cruise Control, International Journal of Vehicle Design, 45(3), p. 339-360, 2007.

[9] J.F. Krems and M.R.K. Baumann, Driving and situation awareness: a cognitive model of memory-update process. Lecture Notes in Computer Science, 2009, Volume 5619/2009, 986-994.

[10] OECD, Behavioural adaptations to changes in the road transport systems. Organization for Economic and Co-operation Development publications, 1990.

[11] S.J. Payne, A descriptive study of mental models, Behaviour \& Information Technology, 1991, Vol. 10, No. 1, 3-21.

[12] PREVENT, Code of Practice for the Design and Evaluation of ADAS. Deliverable D11.2, Integrated Project PREVENT, 2006.

[13] W.B. Rouse and N.M Morris, On looking into the black box: prospects and limits in the search for mental models, Psychological Bulletin 100(3), 349-363, 1986. 
[14]C.M. Rudin-Brown and H.A. Parker, Behavioural adaptation to adaptive cruise control (ACC): implications for preventive strategies. Transportation Research Part F 7 (2004), pp. 59-76.

[15]F. Saad, Some critical issues when studying behavioural adaptations to new driver support systems, Cognitive, Technology \& Work (2006) 8, pp. 175-181.

[16]A. Smiley, Behavioural Adaptation, Safety, and Intelligent Transportation Systems, Transportation Research Record 1724, pp. 47-51, 2000.

[17] N.A. Stanton, M. Young and B. McCaulder, Drive by wire: the case of driver workload and reclaiming control with Adaptive Cruise Control, Safety Science Vol. 27, No. 2/3. pp. 149159, 1997.
[18]N.A. Stanton and M.S. Young, Driver Behaviour with Adaptive Cruise Control, Ergonomics, 48(10), 1294-1313, 2005.

[19] SWOV, SWOV Fact sheet - Advanced Cruise Control (ACC), SWOV, Leidschendam, the Netherlands, 2010.

[20]J.R. Wilson and A. Rutherford, Mental Models: Theory and Application in Human Factors, Human Factors, 1989, 31 (6), 617-634.

[21] World Health Organization, Global Plan for the Decade of Action for Road Safety 2011-2020, World Health Organization publications, 2010 